\title{
SEMICROSSED PRODUCTS OF THE DISK ALGEBRA: CONTRACTIVE REPRESENTATIONS AND MAXIMAL IDEALS
}

\author{
Dale R. Buske and Justin R. Peters
}

\begin{abstract}
Given the disk algebra $\mathcal{A}(\mathbb{D})$ and an automorphism $\alpha$, there is associated a non-self-adjoint operator algebra $\mathbb{Z}^{+} \times_{\alpha} \mathcal{A}(\mathbb{D})$ called the semicrossed product of $\mathcal{A}(\mathbb{D})$ with $\alpha$. We consider those algebras where the automorphism arises via composition with parabolic, hyperbolic, and elliptic conformal maps $\varphi$ of $\mathbb{D}$ onto itself. To characterize the contractive representations of $\mathbb{Z}^{+} \times_{\alpha} \mathcal{A}(\mathbb{D})$, a noncommutative dilation result is obtained. The result states that given a pair of contractions $S, T$ on some Hilbert space $\mathcal{H}$ which satisfy $T S=S \varphi(T)$, there exist unitaries $U, V$ on some Hilbert space $\mathcal{K} \supset \mathcal{H}$ which dilate $S$ and $T$ respectively and satisfy $V U=U \varphi(V)$. It is then shown that there is a one-to-one correspondence between the contractive (and completely contractive) representations of $\mathbb{Z}^{+} \times{ }_{\alpha} \mathcal{A}(\mathbb{D})$ on a Hilbert space $\mathcal{H}$ and pairs of contractions $S$ and $T$ on $\mathcal{H}$ satisfying $T S=S \varphi(T)$. The characters, maximal ideals, and strong radical of $\mathbb{Z}^{+} \times_{\alpha} \mathcal{A}(\mathbb{D})$ are then computed. In the last section, we compare the strong radical to the Jacobson radical.
\end{abstract}

\section{Introduction.}

A semicrossed product of the disk algebra is an operator algebra associated to the pair $(\mathcal{A}(\mathbb{D}), \alpha)$, where $\mathcal{A}(\mathbb{D})$ is the disk algebra and $\alpha$ an automorphism of $\mathcal{A}(\mathbb{D})$. Any such $\alpha$ has the form $\alpha(f)=f \circ \varphi(f \in \mathcal{A}(\mathbb{D}))$ for a linear fractional transformation $\varphi$. It is well-known there is a one-to-one correspondence between contractions (i.e., bounded linear operators $T$ on some Hilbert space with $\|T\| \leq 1$ ) and contractive representations of $\mathcal{A}(\mathbb{D})$. Here, analogously, there is a one-to-one correspondence between pairs $S, T$ of contractions satisfying the relation $T S=S \varphi(T)$ and contractive representations of the semicrossed product, denoted $\mathbb{Z}^{+} \times{ }_{\alpha} \mathcal{A}(\mathbb{D})$, associated to $(\mathcal{A}(\mathbb{D}), \alpha)$. This is meaningful since linear fractional transformations map contractions to contractions (cf. [Sz-NF]). The question of whether contractive representations of $\mathbb{Z}^{+} \times_{\alpha} \mathcal{A}(\mathbb{D})$ can be dilated to representations of the $C^{*}$-crossed product $\mathbb{Z} \times{ }_{\alpha} C(\mathbb{T})$ is equivalent to the following: given a pair of contractions $S, T$ on some Hilbert space $\mathcal{H}$ satisfying $T S=S \varphi(T)$ do 
there exist unitaries $U, V$ on some Hilbert space $\mathcal{K} \supset \mathcal{H}$ which are dilations of $S, T$ respectively, and satisfy $V U=U \varphi(V)$ ? This question, which is of interest in its own right, has an affirmative solution [Theorem II.4]. Furthermore, it marks the starting point in our study of semicrossed products of the disk algebra, by giving faithful representations of $\mathbb{Z}^{+} \times_{\alpha} \mathcal{A}(\mathbb{D})$.

Section $V$ deals with the characterization of the maximal ideal space. The character space, or equivalently the space of maximal ideals of codimension one, was easily obtained from the representation theory [Corollary III.11]. It turns out there are no maximal ideals of codimension greater than one unless the automorphism (i.e., the linear fractional transformation) is elliptic of finite period, say $K$, in which case the maximal ideals have codimension either 1 or $K^{2}$. Finally, these results, together with [HPW] are used to compare the strong radical with the Jacobson radical: the two radicals coincide except when $\alpha$ is elliptic and nonperiodic (that is, an irrational rotation). (Theorem VI.1.)

\section{Dilating Noncommuting Contractions.}

It is well-known that each contraction $T$ on a Hilbert space $\mathcal{H}$ can be dilated to a unitary $U$ on a Hilbert space $\mathcal{K} \subseteq \mathcal{H}$ [Sz-NF]. That is, for $n \geq 1, T^{n} h=P_{\mathcal{H}} U^{n} h \forall h \in \mathcal{H}$. This result then yields the fact that the contractive representations of the disk algebra $\mathcal{A}(\mathbb{D})$ on $\mathcal{H}$ are in a one-to-one correspondence with contractions on $\mathcal{H}[\mathbf{D P}],[\mathbf{S z}-\mathbf{N F}]$. Moreover, it shows that each contractive representation is completely contractive $[\mathbf{D P}]$. Andô then generalized this result by showing that every commuting pair of contractions $S$ and $T$ on $\mathcal{H}$ have a unitary dilation on some $\mathcal{K} \supseteq \mathcal{H}[\mathbf{A}]$. That is, there exist unitaries $U$ and $V$ on $\mathcal{K}$ such that $\forall m \geq 1, n \geq 1, S^{m} T^{n} h=P_{\mathcal{H}} U^{m} V^{n} h$. Hence the contractive representations of the bidisk algebra $\mathcal{A}\left(\mathbb{D}^{2}\right)$ on $\mathcal{H}$ are in one-to-one correspondence with commuting pairs of contractions on $\mathcal{H}$. Furthermore, each representation of $\mathcal{A}\left(\mathbb{D}^{2}\right)$ is completely contractive [DP]. Recently, Sebestyén showed that every anti-commuting pair of contractions have such a dilation $[\mathbf{S}]$. In this section we show that when $\varphi$ is a conformal automorphism of $\mathbb{D}$ and $S$ and $T$ are contractions on $\mathcal{H}$ satisfying $T S=S \varphi(T)$ then a unitary dilation exists. This result is then used to characterize the contractive representations of a semicrossed product. Proofs in this section closely resemble those in $[\mathbf{S}]$. Lemma II.1 is directly lifted from $[\mathbf{S}]$.

Lemma II.1. Let $\mathcal{K}$ and $\mathcal{K}^{\prime}$ be Hilbert spaces, $\mathcal{H} \subset \mathcal{K}$ and $\mathcal{H}^{\prime} \subset \mathcal{K}^{\prime}$ be subspaces and $X: \mathcal{H} \rightarrow \mathcal{K}^{\prime}$ and $X^{\prime}: \mathcal{H}^{\prime} \rightarrow \mathcal{K}$ be given bounded linear transformations. Then, there exists an operator $Y: \mathcal{K} \rightarrow \mathcal{K}^{\prime}$ extending $X$ so that $Y^{*}$ extends $X^{\prime}$ if and only if $\left\langle X h, h^{\prime}\right\rangle=\left\langle h, X^{\prime} h^{\prime}\right\rangle \forall h \in \mathcal{H}, h^{\prime} \in \mathcal{H}^{\prime}$. 
Moreover, $\|Y\| \leq \max \left\{\|X\|,\left\|X^{\prime}\right\|\right\}$.

Suppose now that $S$ and $T$ are contractions on $\mathcal{H}$ which satisfy $T S=$ $S \varphi(T)$ for some linear fractional transformation $\varphi$ of $\mathbb{D}$. Note that $\varphi(T)$ is a well-defined contraction by the functional calculus found in [Sz-NF]. Let $U$ be the minimal isometric dilation of $S$ acting on a Hilbert space $\mathcal{K}$ containing $\mathcal{H}$. Then, $U^{*}$ extends $S^{*}$, where $\mathcal{K}=\bigvee_{n=0}^{\infty} \mathcal{K}_{n}$ and $\mathcal{K}_{n}=\bigvee_{k=0}^{n} U^{k}(\mathcal{H})$.

Lemma II.2. Let $S$ and $T$ be contractions on $\mathcal{H}$ such that $T S=S \varphi(T)$. If $U$ is the minimal isometric dilation of $S$ acting on $\mathcal{K}$, then there exists $T_{\varphi}$ an operator on $\mathcal{K}$ such that $T_{\varphi}^{*}$ extends $T^{*},\left\|T_{\varphi}\right\| \leq 1$, and $T_{\varphi} U=U \varphi\left(T_{\varphi}\right)$.

Proof. The proof is similar to $[\mathbf{S}]$. At the $n$-th step of induction, Lemma II.1 is applied to the maps $\left.U \varphi\left(T_{n-1}\right) U^{*}\right|_{\mathcal{H}_{n-1}}: \mathcal{H}_{n-1} \equiv U\left(\mathcal{K}_{n-1}\right) \rightarrow \mathcal{K}_{n}$ and $T_{n-1}^{*}: \mathcal{K}_{n-1} \rightarrow \mathcal{K}_{n}$ and $\left\|T_{n}\right\| \leq 1$ since $\left\|\varphi\left(T_{n-1}\right)\right\| \leq 1$. Supposing that $\varphi=\mu \varphi_{a}$ where $|\mu|=1$ and $\varphi_{a}(z)=\frac{z-a}{1-\bar{a} z}$, the conditions of Lemma II.1 are satisfied since $\left\langle x,\left[\mu \varphi_{a}\left(T_{n}\right)\right]^{*} y\right\rangle=\left\langle x, \bar{\mu} \varphi_{\bar{a}}\left(T_{n}^{*}\right) y\right\rangle=\left\langle x, \bar{\mu} \varphi_{\bar{a}}\left(T_{n-1}^{*}\right) y\right\rangle=$ $\left\langle x,\left[\mu \varphi_{a}\left(T_{n-1}\right)\right]^{*} y\right\rangle$ for $x \in \mathcal{H}_{n-1}$ and $y \in \mathcal{K}_{n-1}[$ Sz-NF, I.4].

Lemma II.3. Let $S$ and $T$ be contractions on $\mathcal{H}$ such that $T S=S \varphi(T)$. If $U$ is the minimal unitary dilation of $S$ acting on a Hilbert space $\mathcal{K}$, then there exists $T_{\varphi}$ on $\mathcal{K}$ which is a dilation of $T$ with $\left\|T_{\varphi}\right\| \leq 1$ and $T_{\varphi} U=U \varphi\left(T_{\varphi}\right)$.

Proof. Let $U_{+}$be the minimal isometric dilation of $S$ acting on $\mathcal{K}_{+} ; \mathcal{H} \subseteq \mathcal{K}_{+} \subseteq$ $\mathcal{K}$. By Lemma II.2, there exists a dilation $T_{\varphi_{+}}$of $T$ to $\mathcal{K}_{+}$with $\left\|T_{\varphi_{+}}\right\| \leq 1$ and $T_{\varphi+} U_{+}=U_{+} \varphi\left(T_{\varphi+}\right)$. By considering a sequence of polynomials $p_{n} \rightarrow \varphi^{-1}$, it follows from $T_{\varphi+} U_{+}=U_{+} \varphi\left(T_{\varphi+}\right)$ that $\varphi^{-1}\left(T_{\varphi+}\right) U_{+}=U_{+} T_{\varphi+}$. Taking adjoints yields $T_{\varphi+}^{*} U_{+}^{*}=U_{+}^{*} \varphi^{-1}\left(T_{\varphi+}\right)^{*}$. Since $U^{*}$ is the unique minimal isometric dilation of $U_{+}^{*}$ it follows by Lemma II.2 and [Sz-NF, I.4] that there exists an operator $T_{\varphi}^{*}$ on $\mathcal{K}$ such that $T_{\varphi}$ extends $T_{\varphi+},\left\|T_{\varphi}^{*}\right\| \leq 1$, and $T_{\varphi}^{*} U^{*}=U^{*} \varphi^{-1}\left(T_{\varphi}\right)^{*}$. Reasoning as above, $T_{\varphi} U=U \varphi\left(T_{\varphi}\right)$.

Theorem II.4. Let $S$ and $T$ be contractions on $\mathcal{H}$ such that $T S=S \varphi(T)$. Then there exists a pair of unitaries $U$ and $V$ such that $V U=U \varphi(V)$ and $S^{m} T^{n}=\left.P_{\mathcal{H}} U^{m} V^{n}\right|_{\mathcal{H}}$ for every $m, n \in \mathbb{N}$.

Proof. As in [S], let $U_{0}$ be the minimal unitary dilation of $S$ and $T_{0}$ a contractive dilation of $T$ with $T_{0} U_{0}=U_{0} \varphi\left(T_{0}\right)$. Then let $V$ be the minimal unitary dilation of $T_{0}$ and proceed to extend $U_{0}$ to a unitary such that $V U=U \varphi(V)$. The proof follows $[\mathbf{S}]$ after it is shown that $U$ defined on $\mathcal{K}=\bigvee_{-\infty}^{\infty} V^{n}\left(\mathcal{K}_{0}\right)$ by $U\left(V^{n} k_{0}\right)=\varphi^{-1}(V)^{n} U_{0} k_{0}$ is isometric. However, since $\mathrm{V}$ is the minimal unitary dilation of $T_{0}$, it follows that $\varphi^{-1}(V)$ is the minimal unitary dilation of $\varphi^{-1}\left(T_{0}\right)$ [Sz-NF, I.4.3] and so $\left\langle U\left(V^{m} h_{0}\right), U\left(V^{n} k_{0}\right)\right\rangle=$ 


$$
\begin{array}{ll}
\left\langle\varphi^{-1}(V)^{m} U_{0} h_{0}, \varphi^{-1}(V)^{n} U_{0} k_{0}\right\rangle=\left\langle\varphi^{-1}\left(T_{0}\right)^{m} U_{0} h_{0}, \varphi^{-1}\left(T_{0}\right)^{n} U_{0} k_{0}\right\rangle= \\
\left\langle U_{0} T_{0}^{m} h_{0}, U_{0} T_{0}^{n} k_{0}\right\rangle=\left\langle V^{m} h_{0}, V^{n} k_{0}\right\rangle . &
\end{array}
$$

\section{The Semicrossed Product.}

Example III.1. Let $\mathcal{D}$ be an operator algebra and $\alpha$ an automorphism of $\mathcal{D}$. Let $(\rho, \mathcal{K})$ be a contractive representation of $\mathcal{D}$ and let $\mathcal{H}=H^{2}(\mathcal{K})$, the space of square summable elements in $\bigoplus_{0}^{\infty} \mathcal{K}$. Define a contractive representation $\pi$ of $\mathcal{D}$ on $\mathcal{H}$ by $\pi(f)\left(\xi_{0}, \xi_{1}, \xi_{2}, \ldots\right)=\left(\rho(f) \xi_{0}, \rho(\alpha(f)) \xi_{1}, \rho\left(\alpha^{2}(f)\right) \xi_{2}, \ldots\right)$. If $U_{+}$is the unilateral shift on $\mathcal{H}$, then $U_{+} \pi(\alpha(f))=\pi(f) U_{+} \forall f \in \mathcal{D}$. Write $\pi=\widetilde{\rho}$ for the contractive representation constructed above.

Example III.2. The disk algebra $\mathcal{A}(\mathbb{D})$ can be considered as an operator algebra acting (via multiplication) on the Hilbert space $L^{2}(\mathbb{T})$. Let $\alpha$ : $\mathcal{A}(\mathbb{D}) \rightarrow \mathcal{A}(\mathbb{D})$ be an (isometric) automorphism of $\mathcal{A}(\mathbb{D})$ so that $\alpha(f)=$ $f \circ \varphi$ for some conformal mapping $\varphi$ of $\mathbb{D}$. Let $U$ be the forward unilateral shift on $\oplus_{i=0}^{\infty} L^{2}(\mathbb{T}), U\left(\xi_{0}, \xi_{1}, \xi_{2}, \ldots\right)=\left(0, \xi_{0}, \xi_{1}, \ldots\right)$. For $f \in \mathcal{A}(\mathbb{D})$, let $D_{f}$ be the diagonal operator on $\oplus_{i=0}^{\infty} L^{2}(\mathbb{T})$ given by $D_{f}\left(\xi_{0}, \xi_{1}, \xi_{2}, \ldots\right)=$ $\left(f \xi_{0}, \alpha(f) \xi_{1}, \alpha^{2}(f) \xi_{2}, \alpha^{3}(f) \xi_{3}, \ldots\right)$. Then $U D_{\alpha(f)}=D_{f} U \forall f \in \mathcal{A}(\mathbb{D})$. We let $\mathfrak{A}_{\alpha}$ denote the norm closed subalgebra of $B\left(\oplus_{i=0}^{\infty} L^{2}(\mathbb{T})\right)$ generated by $U$ and $D_{f}, f \in \mathcal{A}(\mathbb{D})$.

Note that $U D_{\alpha(f)}=D_{f} U$ so that $\mathfrak{A}_{\alpha}$ is commutative if and only if $\alpha$ (and hence $\varphi$ ) is the identity. Further, we remark that every conformal map of $\mathbb{D}$ onto itself has the form $\varphi(z)=\mu \varphi_{a}(z)$ where $\mu \in \mathbb{C},|\mu|=1$, and $\varphi_{a}(z)=\frac{z-a}{1-\bar{a} z}(a \in \mathbb{D})$. We classify these as hyperbolic, parabolic, or elliptic $[\mathrm{B}]$. In the hyperbolic case, $\varphi_{a}: \overline{\mathbb{D}} \rightarrow \overline{\mathbb{D}}$ has two distinct fixed points which lie on $\partial \mathbb{D}$. If $\varphi_{a}$ is parabolic, it has a unique fixed point lying in $\partial \mathbb{D}$. In the elliptic case, $\varphi_{a}$ has one fixed point in $\mathbb{D}$ (and one outside $\mathbb{D}$ ).

By Lemma 10 of $\left[\mathbf{H P W}\right.$, the study of $\mathfrak{A}_{\alpha}$ can be reduced to three specific cases. If $\alpha$ is hyperbolic, parabolic, or elliptic, we can assume $\varphi$ fixes $\{-1,1\},\{1\}$, or $\{0\}$ respectively.

Example III.3. For $f \in \mathcal{A}(\mathbb{D})$ and $\alpha$ as in Example III.2, the composition operator $C_{\varphi^{-1}}$ and the Toeplitz operator $T_{f}$ on $H^{2}(\mathbb{D})$ satisfy $C_{\varphi^{-1}} T_{\alpha(f)}=$ $T_{f} C_{\varphi^{-1}}$. We define $\mathcal{B}_{\alpha}$ to be the norm closed subalgebra of $B\left(H^{2}(\mathbb{D})\right)$ generated by $C_{\varphi^{-1}}$ and $T_{f}(f \in \mathcal{A}(\mathbb{D}))$ where $\alpha(f)(z)=f(\varphi(z))$.

Definition III.4. Let $\alpha$ be an automorphism of $\mathcal{D}, \rho$ a contractive representation of $\mathcal{D}$ on $\mathcal{H}$, and $V$ a contraction (isometry) on $\mathcal{H}$. We say that $(\rho, V)$ is a contractive (isometric) covariant representation of $(\mathcal{D}, \alpha)$ if $V \rho(\alpha(f))=\rho(f) V \forall f \in \mathcal{D}$. 
Remark III.5. Contractive (isometric) covariant representations of $(\mathcal{D}, \alpha)$ exist as exhibited by the examples above. However, unlike covariant representations of $C^{*}$-algebras, there are isometric covariant representations $(\rho, V)$ satisfying $\rho(\alpha(f)) V=V \rho(f)$ but not $\rho(\alpha(f)) V^{*}=V^{*} \rho(f)$. (Examples III.1 and III.3 satisfy both conditions.)

Example III.6. Let $\alpha$ be an elliptic automorphism of $\mathcal{A}(\mathbb{D})$. Let $\rho$ be a contractive representation of $\mathcal{A}(\mathbb{D})$ on $\ell^{2}(\mathbb{C})$ given by $\rho(z)\left(\xi_{0}, \xi_{1}, \xi_{2}, \ldots\right)=$ $\left(0, \mu \xi_{0}, \mu^{2} \xi_{1}, \mu^{3} \xi_{2}, \ldots\right)$ and $V$ the unilateral shift. Then $\rho(\alpha(f)) V=V \rho(f)$ but $\rho(\alpha(f)) V^{*} \neq V^{*} \rho(f)$.

Consider an automorphism $\alpha$ of $C(\mathbb{T})$ given by composition with the restricted Möbius transformation $\left.\varphi\right|_{\mathbb{T}}$. If $\delta_{n}$ denotes the Kronecker delta on $\mathbb{Z}$, the algebra $\ell^{1}(\mathbb{Z}, C(\mathbb{T}), \alpha)$ consists of all formal sums $\sum_{-\infty}^{\infty} \delta_{n} \otimes f_{n}$ with $f_{n} \in C(\mathbb{T}), \sum_{-\infty}^{\infty}\left\|f_{n}\right\|<\infty$. An adjoint and multiplication can be defined (on simple tensors) by $\left(\delta_{n} \otimes f\right)^{*}=\delta_{-n} \otimes \alpha^{-n}(\bar{f})$ and $\left(\delta_{n} \otimes f\right)\left(\delta_{m} \otimes g\right)=$ $\delta_{n+m} \otimes f \alpha^{n}(g)$.

A multiplication could also be defined by letting $\mathbb{Z}$ act on the left side by $\left(\delta_{n} \otimes f\right)\left(\delta_{m} \otimes g\right)=\delta_{n+m} \otimes \alpha^{m}(f) g$. If the Banach space $\ell^{1}(\mathbb{Z}, C(\mathbb{T}), \alpha)$ is provided with this alternate multiplication, and the adjoint is left unchanged, we obtain a new Banach algebra denoted $\ell^{1}(\mathbb{Z}, C(\mathbb{T}), \alpha)^{o p}$. The Banach algebras $\ell^{1}(\mathbb{Z}, C(\mathbb{T}), \alpha)$ and $\ell^{1}(\mathbb{Z}, C(\mathbb{T}), \alpha)^{o p}$ are isomorphic $[\mathbf{P} 1]$.

Define the Banach algebra $\ell^{1}\left(\mathbb{Z}^{+}, \mathcal{A}(\mathbb{D}), \alpha\right)$ to be the subalgebra of $\ell^{1}(\mathbb{Z}, C(\mathbb{T}), \alpha)^{o p}$ consisting of elements of the form $F=\sum_{n \geq 0} \delta_{n} \otimes f_{n}$ with $f_{n} \in \mathcal{A}(\mathbb{D})$ and $\|F\|_{1}=\sum_{n \geq 0}\left\|f_{n}\right\|<\infty$. Endow $\ell^{1}\left(\mathbb{Z}^{+}, \mathcal{A}(\mathbb{D}), \alpha\right)$ with a multiplication $\left(\delta_{n} \otimes f\right)\left(\delta_{m} \otimes g\right)=\delta_{n+m} \otimes \alpha^{m}(f) g$ so that it is a Banach algebra without adjoint.

If $(\rho, V)$ is a contractive covariant representation of $(\mathcal{A}(\mathbb{D}), \alpha)$ on $\mathcal{H}$, then $\pi: \ell^{1}\left(\mathbb{Z}^{+}, \mathcal{A}(\mathbb{D}), \alpha\right) \rightarrow B(\mathcal{H})$ defined by $\pi\left(\sum_{n \geq 0} \delta_{n} \otimes f_{n}\right)=\sum_{n \geq 0} V^{n} \rho\left(f_{n}\right)$ is a contractive representation. Denote this representation by $\pi=V \times \rho$.

Proposition III.7. The correspondence $(\rho, V) \leftrightarrow V \times \rho$ is a bijection between the collection of contractive covariant representations of $(\mathcal{A}(\mathbb{D}), \alpha)$ and contractive representations of $\ell^{1}\left(\mathbb{Z}^{+}, \mathcal{A}(\mathbb{D}), \alpha\right)$.

Proof. By the preceding remarks, we need only show that $\pi$, a contractive representation of $\ell^{1}\left(\mathbb{Z}^{+}, \mathcal{A}(\mathbb{D}), \alpha\right)$ on $\mathcal{H}$, gives rise to a contractive covariant pair $(\rho, V)$ of $(\mathcal{A}(\mathbb{D}), \alpha)$ and that $\pi=V \times \rho$. Define a contraction $V$ on $\mathcal{H}$ by $V=\pi\left(\delta_{1} \otimes 1\right)$. Define a (contractive) representation $\rho$ of $\mathcal{A}(\mathbb{D})$ by $\rho(f)=\pi\left(\delta_{0} \otimes f\right)$. Then $(\rho, V)$ is a contractive covariant representation of $(\mathcal{A}(\mathbb{D}), \alpha)$ since $\rho(f) V=\pi\left(\delta_{0} \otimes f \cdot \delta_{1} \otimes 1\right)=\pi\left(\delta_{1} \otimes \alpha(f)\right)=\pi\left(\delta_{1} \otimes 1 \cdot \delta_{0} \otimes \alpha(f)\right)=$ $V \rho(\alpha(f))$. To complete the proof, note that $\pi=V \times \rho$ on a dense subset of $\ell^{1}\left(\mathbb{Z}^{+}, \mathcal{A}(\mathbb{D}), \alpha\right)$ and hence everywhere. 
Recall that the crossed product $\mathbb{Z} \times{ }_{\alpha} C(\mathbb{T})$ is the completion of $\ell^{1}(\mathbb{Z}, C(\mathbb{T})$, $\alpha$ ) under the norm $\|F\|=\sup \{\|\pi(F)\|: \pi$ is a contractive representation of $\left.\ell^{1}(\mathbb{Z}, C(\mathbb{T}), \alpha)\right\}$ for $F \in \ell^{1}(\mathbb{Z}, C(\mathbb{T}), \alpha)[\mathrm{MM}]$.

Lemma III.8. The Banach algebra $\ell^{1}\left(\mathbb{Z}^{+}, \mathcal{A}(\mathbb{D}), \alpha\right)$ admits a faithful contractive representation.

Proof. Let $\rho$ be the (faithful) representation of $\mathcal{A}(\mathbb{D})$ on $L^{2}(\mathbb{T})$ given by multiplication. As in Example III.1, $\left(\widetilde{\rho}, U_{+}\right)$is a contractive covariant representation of $(\mathcal{A}(\mathbb{D}), \alpha)$. Thus, $U_{+} \times \widetilde{\rho}$ is a contractive representation of $\ell^{1}\left(\mathbb{Z}^{+}, \mathcal{A}(\mathbb{D}), \alpha\right)$ on $\bigoplus_{0}^{\infty} L^{2}(\mathbb{T})$. Suppose $\left(U_{+} \times \widetilde{\rho}\right)\left(\sum_{n \geq 0} \delta_{n} \otimes f_{n}\right)=0$. Then $\sum_{n \geq 0} U^{n} D_{f_{n}}=0$ in $\mathfrak{A}_{\alpha}$ and hence $\left(\sum_{n \geq 0} U^{n} D_{f_{n}}\right)\left(\xi_{0}, 0,0, \ldots\right)=(0,0,0, \ldots)$ $\forall \xi_{0} \in L^{2}(\mathbb{T})$. It follows that $f_{k} \cdot \xi_{0}=0 \forall \xi_{0} \in L^{2}(\mathbb{T})$. By the faithfulness of $\rho, f_{k} \equiv 0 \forall k \geq 0$. Thus, $U_{+} \times \widetilde{\rho}$ is faithful.

With that motivation, we define an operator enveloping norm on $\ell^{1}\left(\mathbb{Z}^{+}\right.$, $\mathcal{A}(\mathbb{D}), \alpha)$.

Definition III.9. For $F \in \ell^{1}\left(\mathbb{Z}^{+}, \mathcal{A}(\mathbb{D}), \alpha\right)$, set $\|F\|=\sup \{\|(V \times \rho)(F)\|$ : $(\rho, V)$ is a contractive covariant representation of $(\mathcal{A}(\mathbb{D}), \alpha)\}$. Define the semicrossed product of $\mathcal{A}(\mathbb{D})$ with $\alpha$, denoted $\mathbb{Z}^{+} \times_{\alpha} \mathcal{A}(\mathbb{D})$, to be the completion of $\ell^{1}\left(\mathbb{Z}^{+}, \mathcal{A}(\mathbb{D}), \alpha\right)$ with respect to this norm.

Since contractive representations $\rho$ of $\mathcal{A}(\mathbb{D})$ on $\mathcal{H}$ correspond bijectively with contractions on $\mathcal{H}$ [Sz-NF], it follows from Proposition III.7 that there is a bijection between the contractive representations of $\ell^{1}\left(\mathbb{Z}^{+}, \mathcal{A}(\mathbb{D}), \alpha\right)$ and the contractive covariant representations of $(\mathcal{A}(\mathbb{D}), \alpha)$. Since $\ell^{1}\left(\mathbb{Z}^{+}, \mathcal{A}(\mathbb{D}), \alpha\right)$ is dense in $\mathbb{Z}^{+} \times{ }_{\alpha} \mathcal{A}(\mathbb{D})$, the contractive covariant representations of $(\mathcal{A}(\mathbb{D}), \alpha)$ give rise to all contractive representations of $\mathbb{Z}^{+} \times_{\alpha} \mathcal{A}(\mathbb{D})$.

Theorem III.10. The contractive representations of $\mathbb{Z}^{+} \times_{\alpha} \mathcal{A}(\mathbb{D})$ are in a one-to-one corespondence with pairs of contractions $S$ and $T$ satisfying $T S=S \varphi(T)$.

Corollary III.11. The character space of $\mathbb{Z}^{+} \times_{\alpha} \mathcal{A}(\mathbb{D})$ is $\mathcal{M}=\left\{\left(z_{0}, \xi_{0}\right) \in\right.$ $\mathbb{C}^{2}:\left|z_{0}\right| \leq 1,\left|\xi_{0}\right| \leq 1$ and either $\xi_{0}=0$ or $\left.\varphi\left(z_{0}\right)=z_{0}\right\}$.

Proof. Any character $\gamma$ (a contractive representation of $\mathbb{Z}^{+} \times_{\alpha} \mathcal{A}(\mathbb{D})$ on $\mathbb{C}$ ) is determined by a pair $\left(z_{0}, \xi_{0}\right) \in \mathbb{C}^{2}$ satisfying $\left|z_{0}\right| \leq 1,\left|\xi_{0}\right| \leq 1$, and $z_{0} \xi_{0}=$ $\xi_{0} \varphi\left(z_{0}\right)$. However, $z_{0} \xi_{0}=\xi_{0} \varphi\left(z_{0}\right)$ if and only if $\xi_{0}=0$ or $z_{0}$ is a fixed point of $\varphi$.

Proposition III.12. $\mathbb{Z}^{+} \times_{\alpha} \mathcal{A}(\mathbb{D})$ is isomorphic to a non-self-adjoint sub- 
algebra of $\mathbb{Z} \times{ }_{\alpha} C(\mathbb{T})$.

Proof. Since $\ell^{1}\left(\mathbb{Z}^{+}, \mathcal{A}(\mathbb{D}), \alpha\right)$ can be considered to be a subalgebra of $\ell^{1}(\mathbb{Z}$, $C(\mathbb{T}), \gamma)$, there exists an embedding $\imath$ of $\ell^{1}\left(\mathbb{Z}^{+}, \mathcal{A}(\mathbb{D}), \alpha\right)$ into $\mathbb{Z} \times{ }_{\alpha} C(\mathbb{T})$. If, for $F \in \ell^{1}\left(\mathbb{Z}^{+}, \mathcal{A}(\mathbb{D}), \alpha\right),\|F\|=\|\imath(F)\|$, then $\imath$ can be extended to an isometric isomorphism $\widehat{\imath}: \mathbb{Z}^{+} \times{ }_{\alpha} \mathcal{A}(\mathbb{D}) \rightarrow \mathbb{Z} \times{ }_{\alpha} C(\mathbb{T})$ so that

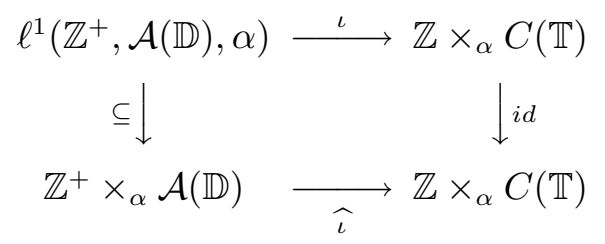

commutes. Since every covariant representation $(\pi, V)$ of $(C(\mathbb{T}), \alpha)$ restricts to a contractive covariant representation of $(\mathcal{A}(\mathbb{D}), \alpha)$, it follows that $\|F\| \geq$ $\|\imath(F)\|$. We show $\|\imath(F)\| \geq\|F\|$ to complete the proof. If $\pi$ is any contractive representation of $\ell^{1}\left(\mathbb{Z}^{+}, \mathcal{A}(\mathbb{D}), \alpha\right)$ on $\mathcal{H}$, it is determined by two contractions $S=\pi\left(\delta_{1} \otimes 1\right)$ and $T=\pi\left(\delta_{0} \otimes z\right)$ which satisfy $T S=S \varphi(T)$. By Theorem II.4, there exist unitaries $U$ and $V$ on $\mathcal{K} \supseteq \mathcal{H}$ such that $V U=U \varphi(V)$ and $S^{m} T^{n}=$ $\left.P_{\mathcal{H}} U^{m} V^{n}\right|_{\mathcal{H}} \forall m, n \in \mathbb{N}$. Then $\pi$ can be extended to a contractive Banach *-representation $\widetilde{\pi}$ of $\ell^{1}(\mathbb{Z}, C(\mathbb{T}), \alpha)^{o p}$ on $\mathcal{K}$ by defining $\widetilde{\pi}\left(\sum_{n=-\infty}^{\infty} \delta_{n} \otimes f_{n}\right)=$ $\sum_{n=-\infty}^{\infty} U^{n} f_{n}(V)$. Hence $\|F\| \leq\|\imath(F)\|$.

Recall that the semicrossed product norm (Definition III.9) was defined by taking a supremum over the collection of contractive covariant representations of $(\mathcal{A}(\mathbb{D}), \alpha)$. By Theorem II.4, we could equally well have defined this norm by taking a supremum over the (smaller) collection of isometric covariant representations of $(\mathcal{A}(\mathbb{D}), \alpha)$. In fact, by Proposition IV.1, we could also have defined this norm by taking a supremum over the pure isometric covariant representations of $(\mathcal{A}(\mathbb{D}), \alpha)$. Moreover, as Corollary III.14 shows, this norm makes every representation of $\mathbb{Z}^{+} \times{ }_{\alpha} \mathcal{A}(\mathbb{D})$ completely contractive.

The proof of the following proposition, which is used only in the subsequent corollary, is left to the reader.

Proposition III.13. The $C^{*}$-envelope of $\mathbb{Z}^{+} \times_{\alpha} \mathcal{A}(\mathbb{D}), C^{*}\left(\mathbb{Z}^{+} \times{ }_{\alpha} \mathcal{A}(\mathbb{D})\right)$, is isometrically isomorphic to $\mathbb{Z} \times{ }_{\alpha} C(\mathbb{T})$.

Corollary III.14. Every contractive representation of $\mathbb{Z}^{+} \times_{\alpha} \mathcal{A}(\mathbb{D})$ is completely contractive.

Proof. By a fundamental theorem of Arveson [Ar], a contractive representation $\rho$ of $\mathbb{Z}^{+} \times{ }_{\alpha} \mathcal{A}(\mathbb{D})$ on $\mathcal{H}$ is completely contractive if and only if there exists a triple $(\mathcal{K}, \widetilde{\rho}, X)$ where $\widetilde{\rho}$ is a $*$-representation of $C^{*}\left(\mathbb{Z}^{+} \times_{\alpha} \mathcal{A}(\mathbb{D})\right)=\mathbb{Z} \times_{\alpha}$ 
$C(\mathbb{T})$ on $\mathcal{K}$ and an isometry $X: \mathcal{H} \rightarrow \mathcal{K}$ such that $\rho(F)=X^{*} \widetilde{\rho}(F) X \forall F \in$ $\mathbb{Z}^{+} \times{ }_{\alpha} \mathcal{A}(\mathbb{D})$. However, each contractive representation $\rho$ of $\mathbb{Z}^{+} \times{ }_{\alpha} \mathcal{A}(\mathbb{D})$ on $\mathcal{H}$ is completely determined by a pair of contractions $S$ and $T$ satisfying $T S=S \varphi(T)$. Let $U$ and $V$ be the pair of unitaries on $\mathcal{K}$ generated by Theorem II.4 and take $X: \mathcal{H} \rightarrow \mathcal{K}$ to be the inclusion map. Define then $\widetilde{\rho}$ on a dense subset of $\mathbb{Z} \times{ }_{\alpha} C(\mathbb{T})$ by $\widetilde{\rho}\left(\sum_{-\infty}^{\infty} \delta_{n} \otimes f_{n}\right)=\sum_{-\infty}^{\infty} U^{n} f_{n}(V)$.

\section{A Concrete Representation.}

Proposition IV.1. $\mathbb{Z}^{+} \times{ }_{\alpha} \mathcal{A}(\mathbb{D})$ is completely isometrically isomorphic to $\mathfrak{A}_{\alpha}$.

Proof. Let $\pi: \mathbb{Z} \times{ }_{\alpha} C(\mathbb{T}) \rightarrow B\left(\oplus_{-\infty}^{\infty} L^{2}(\mathbb{T})\right)$ be defined on the dense subset $\ell^{1}(\mathbb{Z}, C(\mathbb{T}), \alpha)$ by $\sum_{-\infty}^{\infty} \delta_{n} \otimes f_{n} \mapsto \sum_{-\infty}^{\infty} U^{n} M_{f_{n}}$ where $U$ is the bilateral shift and $M_{f}\left(\ldots, \xi_{-1}, \xi_{0}, \xi_{1}, \ldots\right)=\left(\ldots, \alpha^{-1}(f) \xi_{-1}, f \xi_{0}, \alpha(f) \xi_{1}, \ldots\right)$. By $[\mathrm{Pe}, 7.7 .5], \pi$ is an isometry since $\pi(f) \cdot \xi=f \cdot \xi\left(\xi \in L^{2}(\mathbb{T})\right)$ is faithful. In fact, $\pi$ is completely isometric as it is a $*$-homomorphism. Hence $\left.\tilde{\pi} \equiv \pi\right|_{\mathbb{Z}^{+} \times_{\alpha} \mathcal{A}(\mathbb{D})}$ is a complete isometry. Note that $\bigoplus_{0}^{\infty} L^{2}(\mathbb{T})$ is invariant under $\tilde{\pi}\left(\mathbb{Z}^{+} \times_{\alpha} \mathcal{A}(\mathbb{D})\right)$ so that $\tilde{\pi}: \mathbb{Z}^{+} \times_{\alpha} \mathcal{A}(\mathbb{D}) \rightarrow B\left(\bigoplus_{0}^{\infty} L^{2}(\mathbb{T})\right)$ defined on a dense subset by $\tilde{\pi}\left(\sum_{n \geq 0} \delta_{n} \otimes f_{n}\right)=\left.\tilde{\pi}\left(\sum_{n \geq 0} \delta_{n} \otimes f_{n}\right)\right|_{\bigoplus_{0}^{\infty} L^{2}(\mathbb{T})}$ is clearly completely contractive onto its range $\mathfrak{A}_{\alpha}$. It is completely isometric if the induced map $\widehat{\pi}_{k}:\left(\mathbb{Z}^{+} \times_{\alpha} \mathcal{A}(\mathbb{D})\right) \otimes M_{k}(\mathbb{C}) \rightarrow \mathfrak{A}_{\alpha} \otimes M_{k}(\mathbb{C})$ is isometric for all $k \geq 1$. Let $F=\left(F_{i j}\right) \in\left(\mathbb{Z}^{+} \times_{\alpha} \mathcal{A}(\mathbb{D})\right) \otimes M_{k}(\mathbb{C})$. We show that

$$
\|\widehat{\pi}(F)\|=\left\|\left(\widetilde{\pi}\left(F_{i j}\right)\right)\right\|=\left\|\left.\left(\widetilde{\pi}\left(F_{i j}\right)\right)\right|_{1} ^{k}\left(\bigoplus_{0}^{\infty} L^{2}(\mathbb{T})\right)\right\| .
$$

Define

$$
\ell_{N}^{2}\left(L^{2}(\mathbb{T})\right)=\left\{\xi=\left(\xi_{k}\right)_{k=-\infty}^{\infty} \in \bigoplus_{-\infty}^{\infty} L^{2}(\mathbb{T}): \xi_{k}=0 \text { for } k<-N\right\} .
$$

Then each $\ell_{N}^{2}\left(L^{2}(\mathbb{T})\right)$ is invariant under $\widetilde{\pi}\left(\mathbb{Z}^{+} \times_{\alpha} \mathcal{A}(\mathbb{D})\right)$ and $\bigcup_{N \geq 0} \ell_{N}^{2}\left(L^{2}(\mathbb{T})\right)$ is dense in $\bigoplus_{-\infty}^{\infty} L^{2}(\mathbb{T})$. Hence, $\bigcup_{N \geq 0} \bigoplus_{1}^{k} \ell_{N}^{2}\left(L^{2}(\mathbb{T})\right)$ is dense in $\bigoplus_{1}^{k}\left(\bigoplus_{-\infty}^{\infty} L^{2}(\mathbb{T})\right)$. It follows that

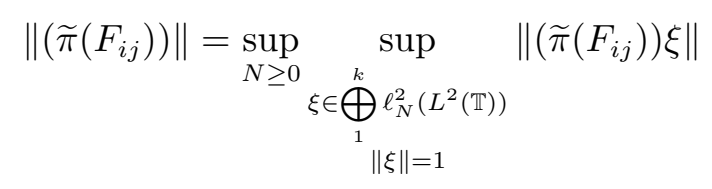




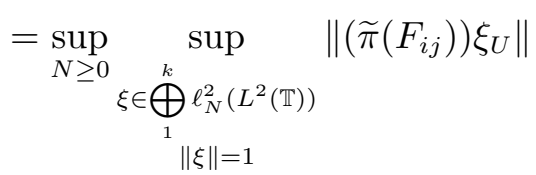

where $\xi_{U}=\left(U^{N} \xi_{1}, U^{N} \xi_{2}, \ldots, U^{N} \xi_{k}\right), U$ is the bilateral shift, and $\xi_{i} \in$ $\ell_{N}^{2} L^{2}(\mathbb{T})$. Thus,

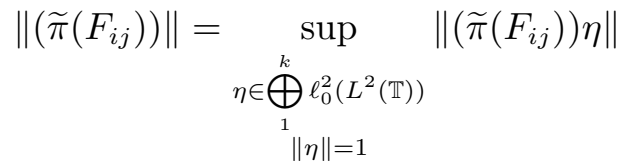

$$
\begin{aligned}
& =\left\|\left.\left(\widetilde{\pi}\left(F_{i j}\right)\right)\right|_{\bigoplus_{1}^{k}\left(\bigoplus_{0}^{\infty} L^{2}(\mathbb{T})\right)}\right\| .
\end{aligned}
$$

Let us now reconsider the algebra $\mathcal{B}_{\alpha}$ defined in Example III.3. In what follows we discuss the isomorphism question regarding $\mathbb{Z}^{+} \times{ }_{\alpha} \mathcal{A}(\mathbb{D})$ and $\mathcal{B}_{\alpha}$ in the case where $\alpha$ is elliptic and irrational. Recall that a change of variables $[$ HPW, Lemma 10] reduces the analysis to the case where $\varphi(z)=\mu z$ where $\mu$ is not a root of unity.

Proposition IV.2. If $\alpha$ is elliptic and nonperiodic, then $\mathfrak{A}_{\alpha}$ is completely isometrically isomorphic to $\mathcal{B}_{\alpha}$.

Proof. Let $\mathcal{C}$ denote the irrational rotation algebra, i.e. the $C^{*}$-algebra generated by any two unitaries $S$ and $T$ satisfying $T S=\mu S T$ [Rf2], [Br]. In particular, $\mathcal{C}$ can be realized as $\mathbb{Z} \times{ }_{\mu} C(\mathbb{T})$ or as the $C^{*}$-algebra of operators on $B\left(L^{2}(\mathbb{T})\right)$ generated by the composition operator $C_{\varphi^{-1}}$ and multiplication operators $M_{f}(f \in C(\mathbb{T}))$. Since $\ell^{1}\left(\mathbb{Z}^{+}, \mathcal{A}(\mathbb{D}), \alpha\right)$ can be isometrically embedded in $\ell^{1}(\mathbb{Z}, C(\mathbb{T}), \mu)$, it follows that $\rho: \mathfrak{A}_{\alpha} \rightarrow \mathcal{C}$ defined on a dense subset by $\rho\left(\sum_{n=0}^{N} U^{n} D_{f_{n}}\right)=\sum_{n=0}^{N} C_{\varphi^{-1}}^{n} M_{f_{n}}$ is an isometric representation on $L^{2}(\mathbb{T})$. Let $\rho_{H^{2}(\mathbb{D})}: \mathfrak{A}_{\alpha} \rightarrow B\left(H^{2}(\mathbb{D})\right)$ be given by $\rho_{H^{2}}(F)=\left.\rho(F)\right|_{H^{2}(\mathbb{D})}$. Then $\rho_{H^{2}}$ is a contractive representation of $\mathfrak{A}_{\alpha}$ onto $\mathcal{B}_{\alpha}$. To show that $\rho_{H^{2}}$ is isometric, we show $\|\rho(F)\|=\left\|\left.\rho(F)\right|_{H^{2}(\mathbb{D})}\right\| \forall F \in \mathfrak{A}_{\alpha}$. This follows as in Proposition IV.1.

By Propositions IV.1 and III.13, $C^{*}\left(\mathfrak{A}_{\alpha}\right) \cong \mathbb{Z} \times{ }_{\alpha} C(\mathbb{T})$. Let $\pi$ be a $C^{*}$ representation of $C^{*}\left(\mathfrak{A}_{\alpha}\right)$ on $L^{2}(\mathbb{T})$ defined by $\pi(U)=C_{\varphi^{-1}}$ and $\pi\left(D_{f}\right)=$ $M_{f}$ where $U$ is the bilateral shift on $\oplus_{-\infty}^{\infty} L^{2}(\mathbb{T})$ and $D_{f}\left(\ldots, \xi_{-1}, \xi_{0}, \xi_{1}, \ldots\right)=$ $\left(\ldots, \alpha^{-1}(f) \xi_{-1}, f \xi_{0}, \alpha(f) \xi_{1}, \ldots\right)$. Let $X: H^{2}(\mathbb{D}) \rightarrow L^{2}(\mathbb{T})$ be inclusion. Then $\rho_{H^{2}}(F)=X^{*} \pi(F) X \forall F \in \mathfrak{A}_{\alpha}$, and $\rho_{H^{2}}$ is completely contractive. 
By the above comments and Proposition III.13, $C^{*}\left(\mathfrak{B}_{\alpha}\right) \cong \mathbb{Z} \times{ }_{\alpha} C(\mathbb{T})$. Let $\pi^{\prime}$ be a $C^{*}$-representation of $C^{*}\left(\mathfrak{B}_{\alpha}\right)$ on $\oplus_{-\infty}^{\infty} L^{2}(\mathbb{T})$ defined by $\pi^{\prime}\left(C_{\varphi^{-1}}\right)=U$ and $\pi^{\prime}\left(M_{f}\right)=D_{f}$ where $U$ and $D_{f}$ are as above. Let $X: \oplus_{0}^{\infty} L^{2}(\mathbb{T}) \rightarrow$ $\oplus_{-\infty}^{\infty} L^{2}(\mathbb{T})$ be inclusion. Then $\rho_{H^{2}}^{-1}(F)=X^{*} \pi^{\prime}(F) X \forall F \in \mathfrak{B}_{\alpha}$ and $\rho_{H^{2}}^{-1}$ is also completely contractive.

When $\alpha$ is elliptic and periodic we can construct a contractive, but not faithful, representation of $\mathfrak{A}_{\alpha}$ onto $\mathcal{B}_{\alpha}$.

Proposition IV.3. $\pi: \mathfrak{A}_{\alpha} \rightarrow \mathcal{B}_{\alpha}$ determined by $U \mapsto C_{\varphi^{-1}}$ and $D_{f} \mapsto T_{f}$ is a contractive, surjective homomorphism.

Proof. The result follows by Proposition IV.1 and the fact that a contractive representation of $\mathbb{Z}^{+} \times{ }_{\alpha} \mathcal{A}(\mathbb{D})$ is completely determined by two contractions $S$ and $T$ satisfying $T S=S \varphi(T)$.

Remark IV.4. Proposition IV.3 shows that algebraically $\mathfrak{A}_{\alpha} / \operatorname{ker} \pi \cong$ $\mathcal{B}_{\alpha}$. However, ker $\pi \neq(0)$. For example, if $f \in \mathcal{A}(\mathbb{D})$ then $0=C_{\varphi^{-1}} T_{f}+$ $C_{\varphi^{-1}}^{K+1} T_{-f}=\pi\left(U D_{f}+U^{K+1} D_{-f}\right)$. This algebraic isomorphism explains the disparity in the character spaces of $\mathfrak{A}_{\alpha}$ and $\mathcal{B}_{\alpha}[\mathbf{H}]$ when $\alpha$ is periodic.

\section{The Maximal Ideal Space.}

In this section we show that the maximal ideal space of $\mathfrak{A}_{\alpha}$ is the same as the character space except in the case where $\alpha$ is elliptic and periodic. We use an ergodic argument for the nonperiodic elliptic case and a spectral argument for the hyperbolic and parabolic cases. We then characterize the maximal ideal space in the case where $\alpha$ is periodic.

Recall from Corollary III.11 that the maps $\gamma_{z_{0}}^{\left(\xi_{0}\right)}: \mathfrak{A}_{\alpha} \rightarrow \mathbb{C}$ (where $\left|z_{0}\right| \leq$ $1,\left|\xi_{0}\right| \leq 1$, and $\varphi\left(z_{0}\right)=z_{0}$ if $\xi_{0} \neq 0$ ) defined on a dense subset by $\gamma_{z_{0}}^{\left(\xi_{0}\right)}\left(\sum_{i=0}^{n} U^{i} D_{f_{i}}\right)=\sum_{i=0}^{n} f_{i}\left(z_{0}\right) \xi_{0}^{i}$ are the characters of $\mathfrak{A}_{\alpha}$. We remark that the multiplicative linear functionals of $\mathfrak{A}_{\alpha}$ could also be calculated by using a technique similar to that found in $[\mathbf{H}]$ and $[\mathbf{H H}]$.

Remark V.I. When $\alpha$ is elliptic and $\beta$ is either parabolic or hyperbolic, it is known that $\mathfrak{A}_{\alpha}$ is not isomorphic to $\mathfrak{A}_{\beta}$. This follows since the radical in the elliptic case is $\{0\}$ whereas in the other cases the radical is the nontrivial set of quasinilpotents (Theorems 11 and 12 of [HPW]). Knowing the characters of $\mathfrak{A}_{\alpha}$ allows us to conclude that $\mathfrak{A}_{\alpha} \neq \mathfrak{A}_{\beta}$ when $\alpha$ is parabolic and $\beta$ is hyperbolic; for if such an isomorphism $\Gamma$ existed, it would induce a homeomorphism $\tau$ of the character spaces defined by $\tau(\gamma)(F)=\gamma(\Gamma(F))$.

To each $F \in \mathfrak{A}_{\alpha}$ we may associate a unique Fourier series, $F \sim$ $\sum_{n=0}^{\infty} U^{n} D_{f_{n}}$. We denote by $\pi_{n}(F)$ the $n$th Fourier coefficient of $F$. Some 
useful properties of these Fourier coefficients are listed in the following lemma from $[\mathbf{H P W}]$.

Lemma V.2. For $n=0,1,2, \ldots$, there is a linear mapping $\pi_{n}: \mathfrak{A}_{\alpha} \rightarrow \mathcal{A}(\mathbb{D})$ satisfying

(i) $\left\|\pi_{n}(F)\right\| \leq\|F\|, F \in \mathfrak{A}_{\alpha}$.

(ii) $\pi_{0}(F G)=\pi_{0}(F) \pi_{0}(G)$ for $F, G \in \mathfrak{A}_{\alpha}$.

(iii) $\pi_{n}\left(\sum_{k=0}^{N} U^{k} D_{f_{k}}\right)=\left\{\begin{array}{cc}f_{n} & 0 \leq n \leq N \\ 0 & n>N\end{array}\right\}$.

(iv) $\pi_{n}(F)=0 \forall n \geq 0 \Rightarrow F \equiv 0$.

Consider the case where $\alpha$ is either parabolic or hyperbolic. From Theorem 12 of $[\mathbf{H P W}]$, the Jacobson radical is $\operatorname{Rad}\left(\mathfrak{A}_{\alpha}\right)=\left\{F \in \mathfrak{A}_{\alpha}: \pi_{0}(F)=0\right.$ and $\pi_{n}(F)\left(z_{0}\right)=0$ for $\left.\varphi\left(z_{0}\right)=z_{0}\right\}$. That is, the radical is precisely the set of quasinilpotent elements. We show by way of contradiction that every maximal ideal $\mathcal{M}$ in $\mathfrak{A}_{\alpha}$ contains the commutator ideal, denoted $\mathcal{C}$, and hence is of codimension one.

Lemma V.3. If $\mathcal{B}$ is a (unital) Banach algebra and $\mathcal{M}$ is a maximal ideal in $\mathcal{B}$ not containing the commutator ideal $\mathcal{C}$, then $\mathcal{B}=\mathcal{M}+\mathcal{C}$.

Proof. By the maximality of $\mathcal{M}$, we can find $b_{0}=m_{0}+c_{0} \in(\mathcal{M}+\mathcal{C}) \cap$ $\left\{b \in \mathcal{B}:\|b-1\|<\frac{1}{2}\right\}$ where $m_{0} \in \mathcal{M}$ and $c_{0} \in \mathcal{C}$. Since $b_{0}$ is invertible, $1=b_{0}^{-1} m_{0}+b_{0}^{-1} c_{0} \in \mathcal{M}+\mathcal{C}$ and hence $\mathcal{B}=\mathcal{M}+\mathcal{C}$.

Proposition V.4. Let $\alpha$ be parabolic or hyperbolic. The maximal ideals of $\mathfrak{A}_{\alpha}$ are precisely the kernels of its characters.

Proof. We show that any maximal ideal $\mathcal{M}$ contains the commutator ideal. Suppose it does not. By the above lemma, $\exists F \in \mathcal{M}$ and $C \in \mathcal{C}$ such that $D_{1}=F+C$. Since $\gamma_{z}^{(0)}(C)=0$ it follows that $\gamma_{z}^{(0)}(F)=1 \forall z \in \overline{\mathbb{D}}$. Write $F=D_{1}+G$ so that $\pi_{0}(G) \equiv 0$ and $\pi_{n}(F) \equiv \pi_{n}(G)$ for $n \geq 1$. Let $z_{0}$ be a fixed point of $\varphi$. Since $\gamma_{z_{0}}^{(\xi)}(C)=0$ it follows that $\gamma_{z_{0}}^{(\xi)}(F)=\gamma_{z_{0}}^{(\xi)}\left(D_{1}\right)=$ $1 \forall \xi \in \overline{\mathbb{D}}$. Hence, $\gamma_{z_{0}}^{(\xi)}\left(F-D_{1}\right)=\sum_{n=1}^{\infty} \pi_{n}(F)\left(z_{0}\right) \xi^{n}=0 \forall \xi \in \overline{\mathbb{D}}$. Thus, $\pi_{n}(F)\left(z_{0}\right)=0$ for $n \geq 1$, and so $\pi_{0}(G) \equiv 0$ and $\pi_{n}(G)\left(z_{0}\right)=0$ for $n \geq 1$. By the preceeding remarks, $G \in \operatorname{Rad}\left(\mathfrak{A}_{\alpha}\right)$. Hence $\operatorname{sp}(G)=\{0\}$ so that $\operatorname{sp}(F)=\operatorname{sp}\left(D_{1}+G\right)=\{1\}$ by the spectral mapping theorem. But then $F \in \mathcal{M}$ is invertible, contradicting the maximality of $\mathcal{M}$.

We now consider the case where $\alpha$ is elliptic. Recall that we are assuming w.l.o.g. that $\alpha(f)=f \circ \varphi$ where $\varphi(z)=\mu z$ for some $|\mu|=1$. The structure of $\mathfrak{A}_{\alpha}$ is closely tied to whether $\mu$ is a root of unity or not. 
By the special structure of $\alpha$ and the definition of $\mathfrak{A}_{\alpha}$, it is an easy calculation to show that $\tilde{\alpha}: \mathfrak{A}_{\alpha} \rightarrow \mathfrak{A}_{\alpha}$ defined on a dense subset by $\tilde{\alpha}\left(\sum_{i=0}^{n} U^{i} D_{f_{i}}\right)$ $=\sum_{i=0}^{n} U^{i} D_{\alpha\left(f_{i}\right)}$ is an isometric automorphism. If $\mu$ is a $K$ th root of unity, define $\#: \mathfrak{A}_{\alpha} \rightarrow \mathfrak{A}_{\alpha}$ by $F \mapsto \frac{1}{K} \sum_{k=0}^{K-1} \tilde{\alpha}^{k}(F)$. Note that \# is $\tilde{\alpha}$-invariant. Defining $\mathfrak{A}_{0}$ to be the closed subalgebra of $\mathfrak{A}_{\alpha}$ generated by $\left\{U, D_{f}: f\right.$ is $\alpha$-invariant $\}$, it is easy to verify that $\mathfrak{A}_{0}$ is maximal abelian and \# is a linear projection onto $\mathfrak{A}_{0}$. As in $[\mathbf{P} 2, \mathrm{~V} .8]$ we can define a map \# with similar properties when $\alpha$ is nonperiodic by \# $\left(\sum_{i=0}^{n} U^{i} D_{f_{i}}\right) \equiv \sum_{i=0}^{n} U^{i} D_{\int_{\mathbb{T}} f_{i} d m(z)}=$ $\sum_{i=0}^{n} U^{i} D_{f_{i}(0)}$.

Proposition V.5. If $\alpha$ is nonperiodic, then $\mathfrak{A}_{0}$ is the subalgebra of $\mathfrak{A}_{\alpha}$ generated by $\left\{U, D_{1}\right\}$. Furthermore, \# is a linear projection onto the maximal abelian subalgebra $\mathfrak{A}_{0}$ of $\mathfrak{A}_{\alpha}$.

Proof. Let $F=\sum_{i=0}^{n} U^{i} D_{f_{i}} \in \mathfrak{A}_{0}$. Then by $\alpha$-invariance $f_{i}\left(\mu^{k} \cdot \frac{1}{2}\right)=f_{i}\left(\frac{1}{2}\right)$ $\forall k \in \mathbb{N}, 0 \leq i \leq n$, so that analyticity, nonperiodicity of $\alpha$, and the ergodic theorem gives $f_{i} \equiv f_{i}(0)$ on $\mathbb{D}$ and hence $\overline{\mathbb{D}}$. Since \# is clearly a linear projection onto $\mathfrak{A}_{0}$, we need only show $\mathfrak{A}_{0}$ is a maximal abelian subalgebra of $\mathfrak{A}_{\alpha}$. By definition, $\mathfrak{A}_{0}$ is commutative. Suppose that $F \in \mathfrak{A}_{\alpha}, F \sim$ $\sum_{n=0}^{\infty} U^{n} D_{f_{n}}$, commutes with $\mathfrak{A}_{0}$. Then $F U=U F$ and $\alpha\left(f_{n}\right)=f_{n} \forall n \geq 0$. Each $f_{n}$ is then constant by the nonperiodicity of $\alpha$.

The characters of $\mathfrak{A}_{0}$, which are easy to compute, will be used to characterize the maximal ideals in $\mathfrak{A}_{\alpha}$. Since $\mathfrak{A}_{0}$ is a commutative Banach algebra, its maximal ideal space corresponds in a one-to-one fashion with the kernels of its characters. If $\alpha$ is nonperiodic, then $\mathfrak{A}_{0} \cong \mathcal{A}(\mathbb{D})$ (given by $U \mapsto z$ ) and its characters are determined by $U \mapsto \xi \in \overline{\mathbb{D}}$. Denote these by $\gamma_{\mathfrak{A}_{0}}^{(\xi)}$. If $\alpha$ is periodic with period $K$, there are more characters. In fact, if we denote by $\gamma_{\mathfrak{A}_{0}, z_{0}}^{(\xi)}$ the map determined by $U \mapsto \xi$ and $D_{z} \mapsto z_{0}$, the maximal ideal space of $\mathfrak{A}_{0}$ can be computed as the characters of $\mathfrak{A}_{\alpha}$ were using the technique found in $[\mathbf{H}]$ and $[\mathbf{H H}]$. If $\psi=\min \left\{\theta: e^{i \theta}=\mu^{k}, 0 \leq k \leq K-1,0<\theta<2 \pi\right\}$, then $\mathcal{M}_{\mathfrak{A}_{0}}=\left\{\gamma_{\mathfrak{A}_{0}, r e^{i \theta_{0}}}^{(\xi)}: 0 \leq r \leq 1,|\xi| \leq 1,0 \leq \theta_{0}<\psi\right\}$ is the set of characters on $\mathfrak{A}_{0}$.

As in $\left[\mathbf{P} 2\right.$, V.9], for an ideal $\mathcal{I} \subseteq \mathfrak{A}_{0}$ define $\widetilde{\mathcal{I}}=\left\{F \in \mathfrak{A}_{\alpha}: \#\left(H \tilde{\alpha}^{n}(F) G\right) \in\right.$ $\left.\mathcal{I} \forall H, G \in \mathfrak{A}_{\alpha}, n \geq 0\right\}$. Using \# we can then construct a one-to-one correspondence between the maximal ideals in $\mathfrak{A}_{0}$ and the maximal $\tilde{\alpha}$-invariant ideals in $\mathfrak{A}_{\alpha}$.

Proposition V.6. (i) If $\mathcal{M}_{0} \subseteq \mathfrak{A}_{0}$ is a maximal ideal, then $\widetilde{\mathcal{M}}_{0} \subseteq \mathfrak{A}_{\alpha}$ is a maximal $\tilde{\alpha}$-invariant ideal in $\mathfrak{A}_{\alpha}$.

(ii) If $\mathcal{R}$ is a maximal $\tilde{\alpha}$-invariant ideal, then $\#(\mathcal{R}) \subseteq \mathfrak{A}_{0}$ is a maximal 
ideal. Furthermore, $\mathcal{R}=\widetilde{\#(\mathcal{R})}$.

Let $\mathcal{M}$ be a maximal ideal in $\mathfrak{A}_{\alpha}$ Let $\langle U\rangle$ denote the closed ideal in $\mathfrak{A}_{\alpha}$ generated by $U$. Then, $\mathcal{M} \cdot\langle U\rangle \subseteq \tilde{\alpha}(\mathcal{M})$. Furthermore, $\tilde{\alpha}(\mathcal{M})$ maximal implies $\tilde{\alpha}(\mathcal{M})$ is prime so that either $\langle U\rangle \subseteq \tilde{\alpha}(\mathcal{M})$ or $\mathcal{M} \subseteq \tilde{\alpha}(\mathcal{M})$.

Theorem V.7. If $\alpha$ is nonperiodic, the maximal ideal space of $\mathfrak{A}_{\alpha}$ is precisely the space of characters.

Proof. Let $\mathcal{M}$ be maximal in $\mathfrak{A}_{\alpha}$. If $\mathcal{M} \subseteq \tilde{\alpha}(\mathcal{M})$ then $\mathcal{M}=\tilde{\alpha}(\mathcal{M})$. Thus, $\mathcal{M}$ is $\tilde{\alpha}$-invariant and $\mathcal{M}=\widetilde{\#(\mathcal{M})}=\left\{F \in \mathfrak{A}_{\alpha}: \#\left(H \tilde{\alpha}^{n}(F) G\right) \in \operatorname{ker} \gamma \forall n \geq 0\right.$ and $\left.H, G \in \mathfrak{A}_{\alpha}\right\}$ for some $\gamma$ a character on $\mathfrak{A}_{0}$. To show that $\mathcal{M}=\operatorname{ker} \gamma_{0}^{(\xi)}$ for some $\xi \in \overline{\mathbb{D}}$, we need only show $\mathcal{M} \subseteq \operatorname{ker} \gamma_{0}^{(\xi)}$. But $F \sim \sum_{n=0}^{\infty} U^{n} D_{f_{n}}, F \in \mathcal{M}$ implies $0=\gamma(\#(F))=\gamma\left(\sum_{n=0}^{\infty} U^{n} D_{f_{n}(0)}\right)=\sum_{n=0}^{\infty} f_{n}(0) \xi^{n}=\gamma_{0}^{(\xi)}(F)$ for some $\xi \in \overline{\mathbb{D}}$.

If $\langle U\rangle \subseteq \tilde{\alpha}(\mathcal{M})$, then by applying $\widetilde{\alpha}^{-1}$ it follows that $\langle U\rangle \subseteq \mathcal{M} . \mathcal{M} /\langle U\rangle$ is then a maximal ideal in $\mathfrak{A}_{\alpha} /\langle U\rangle$. But $\mathfrak{A}_{\alpha} /\langle U\rangle \cong \mathcal{A}(\mathbb{D})$. Hence, $\mathcal{M} /\langle U\rangle$ corresponds to a maximal ideal in $\mathcal{A}(\mathbb{D})$; namely a kernel of point evaluation. So, $\mathcal{M} /\langle U\rangle=\operatorname{ker} \gamma_{z}^{(0)}$ for some $z \in \overline{\mathbb{D}}$.

We now show that if $\alpha$ has period $K$, there are maximal ideals in $\mathfrak{A}_{\alpha}$ of codimension 1 and $K^{2}$. Define $S_{K}$ to be the $K \times K$ shift matrix given by $S_{i j}=1$ if $i-j=1 \bmod K$ and 0 otherwise and $T(f, \mu)$ to be the $K \times K$ diagonal matrix given by $T(f, \mu)_{j, j}=f\left(\mu^{j-1} z_{0}\right)$. For $\left|w_{0}\right| \leq 1$ and $\left|z_{0}\right| \leq 1$, define $\rho_{z_{0}, w_{0}}: \mathfrak{A}_{\alpha} \rightarrow \mathcal{M}_{K}(\mathbb{C})$ on a dense subset by $\sum_{\ell=0}^{K L-1} \overline{U^{\ell}} D_{f_{\ell}} \mapsto$ $\sum_{\ell=0}^{K L-1} w_{0}^{\ell} S_{K}^{\ell} T\left(f_{\ell}, \mu\right)$.

Lemma V.8. If $\left|z_{0}\right| \leq 1,\left|w_{0}\right| \leq 1$, then $\rho_{z_{0}, w_{0}}$ is a contractive representation.

Proof. This follows by Theorem III.10 since $\rho_{z_{0}, w_{0}}$ is determined by two contractions $S_{K}$ and $T(z, \mu)$ satisfying $T(z, \mu) S_{K}=S_{K} \varphi(T(z, \mu))$.

By the simplicity of $\mathcal{M}_{K}(\mathbb{C}), \operatorname{ker} \rho_{z_{0}, w_{0}}$ is a maximal ideal in $\mathfrak{A}_{\alpha}$ if $z_{0} \neq 0$ and $w_{0} \neq 0$.

Lemma V.9. If $z_{0} \neq 0$ and $w_{0} \neq 0$, then $\rho_{z_{0}, w_{0}}$ is a contractive representation of $\mathfrak{A}_{\alpha}$ onto $\mathcal{M}_{K}(\mathbb{C})$.

Proof. We need only show that if $z_{0} \neq 0$ and $w_{0} \neq 0$, then $\rho_{z_{0}, w_{0}}$ is onto. For $0 \leq i, j \leq K-1$, define

$$
f_{i, j}(z)=\frac{1}{w_{0}^{K+i-j(\bmod K)}} \cdot \frac{\prod_{\substack{l=0 \\ l \neq K-i}}^{K-1}\left(\mu^{l} z-z_{0}\right)}{\prod_{l=1}^{K-1}\left(\mu^{l} z_{0}-z_{0}\right)} .
$$


Then, for $0 \leq k \leq K-1$,

$$
\begin{aligned}
f_{i, j}\left(\mu^{k} z_{0}\right) & =\frac{1}{w_{0}^{K i-u(\bmod K)}} \cdot \frac{\prod_{\substack{l=0 \\
l \neq-i(\bmod K)}}^{K-1}\left(\mu^{k+l} z_{0}-z_{0}\right)}{\prod_{l=1}^{K-1}\left(\mu^{l} z_{0}-z_{0}\right)} \\
& =\frac{1}{w_{0}^{K+i-j(\bmod K)}}\left\{\begin{array}{ccc}
0 & \text { if } & k \neq i \\
1 & \text { if } & k=i
\end{array}\right\} \\
& =\frac{1}{w_{0}^{K+i-j(\bmod K)}} \delta_{k, i} .
\end{aligned}
$$

Hence, $\rho_{z_{0}, w_{0}}$ is onto $\mathcal{M}_{K}(\mathbb{C})$ as $E_{i j}=\rho_{z_{0}, w_{0}}\left(U^{K+i-j(\bmod K)} D_{f_{i, j}}\right)$.

Theorem V.10. If $\alpha$ has period $K$ and $\mathcal{M}$ is a maximal ideal in $\mathfrak{A}_{\alpha}$, then $\mathcal{M}=\operatorname{ker} \rho_{z_{0}, w_{0}}$ for some $z_{0}, w_{0} \in \overline{\mathbb{D}}$.

Proof. As in the nonperiodic case, $\tilde{\alpha}(\mathcal{M})$ is maximal and hence prime with either $\mathcal{M} \subseteq \tilde{\alpha}(\mathcal{M})$ or $\langle U\rangle \subseteq \tilde{\alpha}(\mathcal{M})$. If $\langle U\rangle \subseteq \tilde{\alpha}(\mathcal{M})$, then since $\langle U\rangle$ is $\tilde{\alpha}$-invariant and $\alpha$ is periodic, $\langle U\rangle \subseteq \tilde{\alpha}^{K}(\mathcal{M})=\mathcal{M}$. Thus, $\mathcal{M}=\operatorname{ker} \rho_{z_{0}, 0}$ for some $z_{0} \in \overline{\mathbb{D}}$. Suppose then $\mathcal{M} \subseteq \tilde{\alpha}(\mathcal{M})$ so that $\mathcal{M}=\tilde{\alpha}(\mathcal{M})$. By Proposition V.6, \#(M) $=\operatorname{ker} \gamma$ for some character $\gamma$ on $\mathfrak{A}_{0}$ and hence $\mathcal{M}=$ $\widetilde{\#(\mathcal{M})}=\widehat{\operatorname{ker} \gamma_{\mathfrak{A}_{0}, z_{0}^{\prime}}^{\left(\xi^{\prime}\right)}}$ for some $\xi^{\prime} \in \overline{\mathbb{D}}$ and $z_{0}^{\prime}=r e^{i \theta}$ where $0 \leq r \leq 1,0 \leq$ $\theta<\psi$, and $\psi=\min \left\{\theta: e^{i \theta}=\mu^{k}, 0 \leq k \leq K-1,0<\theta<2 \pi\right\}$. Since $\operatorname{ker} \rho_{z_{0}, w_{0}}$ is maximal in $\mathfrak{A}_{\alpha}$, we need only show that $\operatorname{ker} \rho_{z_{0}, w_{0}} \subseteq \mathcal{M}$ for some $z_{0}, w_{0}$. But, $\operatorname{ker} \rho_{z_{0}^{\prime}, \xi^{\prime}}$ is $\tilde{\alpha}$-invariant so that $\rho_{z_{0}^{\prime}, \xi^{\prime}}\left(\tilde{\alpha}^{n}(F)\right)=0 \forall n \geq 0$ and $F \in \operatorname{ker} \rho_{z_{0}^{\prime}, \xi^{\prime}}$. Hence $\rho_{z_{0}^{\prime}, \xi^{\prime}}\left(\#\left(H \tilde{\alpha}^{n}(F) G\right)\right)=0 \forall n \geq 0$ and $H, G \in \mathfrak{A}_{\alpha}$ yielding $\gamma_{z_{0}^{\prime}}^{\left(\xi^{\prime}\right)}\left(\#\left(H \tilde{\alpha}^{n}(F) G\right)\right)=0$ and $F \in \mathcal{M}$.

\section{VI. The Strong Radical.}

Having computed the maximal ideal space of $\mathfrak{A}_{\alpha}$, we can now compute its strong radical and compare it to its Jacobson radical. For the remainder $\alpha$ will be fixed.

Theorem VI.1. Let $\mathfrak{A}_{J}$ and $\mathfrak{A}_{S}$ denote the Jacobson and strong radicals of $\mathfrak{A}_{\alpha}$ respectively.

(i) If $\alpha$ is parabolic or hyperbolic, $\mathfrak{A}_{J}=\mathfrak{A}_{S}$.

(ii) If $\alpha$ is elliptic and nonperiodic, $\mathfrak{A}_{J} \subsetneq \mathfrak{A}_{S}$.

(iii) If $\alpha$ is elliptic and periodic, $\mathfrak{A}_{J}=\mathfrak{A}_{S}=(0)$. 
Proof. From $[\mathrm{HPW}$, the Jacobson radical is precisely the set of quasinilpotents. If $\alpha$ is parabolic or hyperbolic, then $\mathfrak{A}_{J}=\left\{F \in \mathfrak{A}: \pi_{0}(F) \equiv 0\right.$ and $\pi_{n}(F)\left(z_{0}\right)=0 \forall n \geq 1$ for $z_{0}$ fixed by $\left.\alpha\right\}$. Since the maximal ideals are precisely the kernels of the characters in these cases, (i) follows as

$$
\begin{aligned}
\mathfrak{A}_{S} & =\left\{F \in \mathfrak{A}_{\alpha}: F \in \operatorname{ker} \gamma_{z}^{(0)} \forall z \in \overline{\mathbb{D}} \text { and } F \in \operatorname{ker} \gamma_{z_{0}}^{(\xi)} \forall \xi \in \overline{\mathbb{D}}\right. \\
& \text { for } \left.z_{0} \text { fixed by } \alpha\right\} \\
& =\left\{F: \pi_{0}(F) \equiv 0 \text { and } \sum_{n \geq 1} \pi_{n}(F)\left(z_{0}\right) \xi^{n}=0 \forall \xi \in \overline{\mathbb{D}}\right\} \\
& =\left\{F: \pi_{0}(F) \equiv 0 \text { and } \pi_{n}(F)\left(z_{0}\right)=0 \forall n \geq 1 \text { for } z_{0} \text { fixed by } \alpha\right\} \\
& =\mathfrak{A}_{J} .
\end{aligned}
$$

If $\alpha$ is elliptic, $\mathfrak{A}_{J}=(0)$. When $\alpha$ is nonperiodic, $\mathfrak{A}_{S} \supsetneq(0)$ as $U D_{z} \in \mathfrak{A}_{S}$ for example. In fact, $\mathfrak{A}_{S}=\left\{F: \pi_{0}(F) \equiv 0\right.$ and $\left.\pi_{n}(F)(0)=0 \forall n \geq 1\right\}$. If $\alpha$ is periodic of period $K$, we show $\mathfrak{A}_{S}=(0)$ to complete the proof.

First, note that the Fourier series of $F \in \mathfrak{A}_{\alpha}$ is Cesàro summable [P2]. Hence,

$$
\lim _{N \rightarrow \infty}\left\|\sum_{l=0}^{K N-1} \frac{1}{K N}\left(\sum_{m=0}^{l} U^{m} D_{\pi_{m}(F)}\right)-F\right\|=0 .
$$

Let $F \in \mathfrak{A}_{S}$ and $\varepsilon>0$ be given. We show that $\pi_{l}(F) \equiv 0 \forall l \geq 0$ so that $F=0$. Choose $M$ such that if $N \geq M$ we have

$$
\left\|\sum_{l=0}^{K N-1} \frac{1}{K N}\left(\sum_{m=0}^{l} U^{m} D_{\pi_{m}(F)}\right)-F\right\|=\left\|\sum_{l=0}^{K N-1} U^{l} D_{\left(1-\frac{l}{K N}\right) \pi_{l}(F)}-F\right\|<\varepsilon .
$$

Then,

$$
\left\|\rho_{z_{0}, w}\left(\sum_{l=0}^{K N-1} U^{l} D_{\left(1-\frac{l}{K N}\right) \pi_{l}(F)}-F\right)\right\|<\varepsilon \forall z_{0}, w \in \overline{\mathbb{D}} \text { by Lemma V.8. }
$$

Since $F \in \mathfrak{A}_{S}$,

$$
\left\|\rho_{z_{0}, w}\left(\sum_{l=0}^{K N-1} U^{l} D_{\left(1-\frac{l}{K N}\right) \pi_{l}(F)}\right)\right\|<\varepsilon .
$$

In particular, for $0 \leq k \leq K-1$ we have

$$
\left|\sum_{l=0}^{N-1}\left(1-\frac{K l+k}{K N}\right) \pi_{K l+k}(F)\left(z_{0}\right) w^{K l+k}\right|<\varepsilon \forall z_{0} \in \overline{\mathbb{D}}, w \in \mathbb{T} .
$$

Fix $l_{0} \geq 0$. Note that

$$
\int_{\mathbb{T}}\left|\sum_{l=0}^{N-1}\left(1-\frac{K l+k}{K N}\right) \pi_{K l+k}(F)\left(z_{0}\right) w^{K l+k}\right| d m(w)<\varepsilon \forall z_{0} \in \overline{\mathbb{D}} .
$$


It follows, since $\int_{\mathbb{T}} z^{l} d m(z)=0$ unless $l=-1$, that

$$
\begin{aligned}
& \left|1-\frac{K l_{0}+k}{K N}\right|\left|\pi_{K l_{0}+k}(F)\left(z_{0}\right)\right|\left|\int_{\mathbb{T}} w^{-1} d m(w)\right| \\
& =\left|\int_{\mathbb{T}}\left(1-\frac{K l_{0}+k}{K N}\right) \pi_{K l_{0}+k}(F)\left(z_{0}\right) w^{-1} d m(w)\right| \\
& =\left|\int_{\mathbb{T}} \sum_{l=0}^{N-1}\left(1-\frac{(K l+k)}{K N}\right) \pi_{K l+k}(F)\left(z_{0}\right) w^{K\left(l-l_{0}\right)-1} d m(w)\right| \\
& \leq \int_{\mathbb{T}}\left|\sum_{l=0}^{N-1}\left(1-\frac{(K l+k)}{K N}\right) \pi_{K l+k}(F)\left(z_{0}\right) w^{K l+k}\right|\left|w^{-K l_{0}-k-1}\right| d m(w) \\
& =\int_{\mathbb{T}}\left|\sum_{l=0}^{N-1}\left(1-\frac{(K l+k)}{K N}\right) \pi_{K l+k}(F)\left(z_{0}\right) w^{K l+k}\right| d m(w) \\
& <\varepsilon \forall z_{0} \in \overline{\mathbb{D}} .
\end{aligned}
$$

Choosing $N \geq M$ large enough so that $\frac{K l_{0}+k}{K N}<\frac{1}{2}$ it follows that $\left|\pi_{K l_{0}+k}(F)\left(z_{0}\right)\right|$ is arbitrarily small $\forall z_{0} \in \overline{\mathbb{D}}$ so that $\pi_{K l_{0}+k}(F) \equiv 0$ for $0 \leq k \leq K-1$ and hence $F=0$.

Note added in proof (June 1997). Since this paper was submitted we have learned that a proof of Corollary III.14 has been found independently by S.C. Power [Po2].

\section{References}

[A] T. Andô, On a pair of commutative contractions, Acta Sci. Math., 24 (1963), 88-90.

[Ar] W.B. Arveson, Subalgebras of $C^{*}$-algebras, Acta Math., 123 (1969), 141-224.

[Br] B. Brenken, Representations and automorphisms of the irrational rotation algebra, Pacific J. Math., 111 (1984), 257-282.

[B] R.B. Burkel, Iterating analytic self-maps of the disk, Monthly, 88(6) (1981), 396407.

[Bus] D. Buske, Hilbert modules over semicrossed products of the disk algebra, Dissertation, Iowa State University, Ames, 1997.

[DMP] R.G. Douglas, P.S. Muhly and Carl Pearcy, Lifting commuting operators, Michigan Math. J., 15 (1968), 385-395.

[DP] R.G. Douglas and V. Paulsen, Hilbert modules over function algebras, Pitman Longman, 1989.

[HH] D. Hadwin and T. Hoover, Operator algebras and the conjugacy of transformations, J. Funct. Anal., 77 (1988), 112-122. 
[Ho] K. Hoffman, Banach spaces of analytic functions, Prentice-Hall, Englewood Cliffs, NJ, 1962.

[H] T. Hoover, Isomorphic operator algebras and conjugate inner functions, Mich. J. Math., 39 (1992), 229-237.

[HPW] T. Hoover, J. Peters and W. Wogen, Spectral properties of semicrossed products, Houston J. Math., 79(4) (1993), 649-660.

[MM] M.J. McAsey and P.S. Muhly, Representations of non-self-adjoint crossed products, Proc. London Math. Soc., 47(3) (1983), 128-144.

[Pe] G. Pederson, $C^{*}$-algebras and their automorphism groups, Academic Press, London/New York, 1979.

[P1] J. Peters, Semi-crossed products of $C^{*}$-algebras, J. Funct. Anal., 59 (1984), 498-534.

[P2] - The ideal structure of certain nonselfadjoint operator algebras, Trans. Amer. Math. Soc., 305(1) (1988), 333-352.

[P3] Invertibility and topological stable rank for semi-crossed product algebras, Rocky Mountain J. of Math., 20(2) (1990), 511-526.

[Po1] S.C. Power, Classification of analytic crossed product algebras, Bull. London Math. Soc., 24 (1992), 368-372.

[Po2] - Completely contractive representations for some doubly generated antisymmetric operator algebras, to appear.

[Rf1] M.A. Rieffel, Irrational rotation $C^{*}$-algebras, 1978, Abstacts of Short Communication, International Congress of Mathematicians.

[Rf2] $\quad C^{*}$-algebras associated with irrational rotations, Pacific J. Math., 93 (1981), 415-429.

[S] Z. Sebestyén, Anticommutant lifting and anticommuting dilation, Proc. Amer. Math. Soc., 121 (1994), 133-136.

[Sz-NF] B.Sz. Nagy and C. Foias, Harmonic analysis of operators on Hilbert space, NorthHolland, Amsterdam, 1970.

[Wa] P. Walters, Ergodic theory-introductory lectures, Lect. notes in Math., 458, Springer, 1975 .

Received December 21, 1996 and revised July 30, 1997. The second author was partially supported by a grant from National Science Foundation.

St. Cloud State University

St. Cloud, MN 56301

E-mail address: DBuske@stcloudstate.edu

IOWA StATE UNIVERSity

AmEs, IA 50011

E-mail address: peters@iastate.edu 